\title{
EL SENTIDO DE PERTENENCIA, UNA ESTRATEGIA DE MEJORA EN EL PROCESO FORMATIVO EN LAS ARTES. ESTUDIO DE CASO EN DANZA EN UNA UNIVERSIDAD MEXICANA
}

\author{
The Sense of Belonging, a Strategy of Improvement in the Training Process in the Arts. \\ Case Study in Dance at a Mexican University
}

\author{
Alhelí Corona ${ }^{1}$ \\ ORCID: 0000-0002-1398-5377 \\ ${ }^{1}$ Benemérita Universidad Autónoma de Puebla, México. \\ Correo: jaesya@gmail.com
}

Recibido: $26 / 08 / 2020$

Aceptado: 05/10/2020

Resumen: El sentido de pertenencia es un sentimiento de identidad que el individuo genera con la comunidad con la que interactúa para alcanzar metas en común. Las artes, a su vez, demandan consolidar una identidad y difundir experiencias artísticas en las aulas. Por tal motivo, este estudio tiene como objetivo conocer cómo se presenta el sentido de pertenencia desde la opinión estudiantil en la Licenciatura en Danza de la Benemérita Universidad Autónoma de Puebla (BUAP), México. Se condujo un estudio cuantitativo a través de un cuestionario que valoró el sentido de pertenencia en cuatro dimensiones (psicológica-social, afectiva, académica y física). Los resultados apuntan que las dimensiones académica y social requieren acciones encaminadas a la autoconfianza y al acercamiento con el medio laboral. Analizar el sentido de pertenencia permite la construcción de significantes comunes que fortalezcan la formación profesional del estudiantado.

Palabras clave: sentido de pertenencia; educación superior; danza; artes.

\begin{abstract}
The sense of belonging is a feeling of identity that individuals generate with the community with which they interact to achieve common goals. The arts, in turn, demand the consolidation of an identity and the dissemination of artistic experiences in the classroom. For this reason, this study aims to find out how the sense of belonging is presented from the student's point of view in the Dance Degree of the Benemérita Universidad Autónoma de Puebla (BUAP), Mexico. A quantitative study was conducted through a questionnaire that evaluated the sense of belonging in four dimensions (psychological-social, affective, academic and physical). The results indicate that the academic and social dimensions require actions aimed at self-confidence and approaching the work environment. Analyzing the sense of belonging allows the construction of common meanings that strengthen the professional formation of the students.
\end{abstract}

Keywords: sense of belonging; higher education; dance; arts. 


\section{Introducción: la incorporación de la investigación educativa en las artes}

Las instituciones de Educación Superior (IES) que ofertan licenciaturas en danza adquieren una gran responsabilidad con la sociedad comprometiéndose a generar, promover y difundir reflexiones artísticas, culturales, sociales, políticas y económicas donde el cuerpo y la mente se conjugan para generar una experiencia estética en los otros. Para lograr que estas experiencias estéticas sean transmitidas a la sociedad, las IES deben dotar a los estudiantes de conocimientos y habilidades que les permitan desarrollar competencias en diferentes técnicas, estilos dancísticos, producción escénica, gestión y creación de propuestas innovadoras a través de la danza y el movimiento con la finalidad de influir en la sensibilidad social.

La demanda de formación integral para alumnos en las universidades es una tarea que crece rápidamente y que muchas veces resulta difícil atender por la complejidad que esto conlleva. En el caso de las artes el compromiso se incrementa, ya que los estudiantes y profesionales de la danza se encargan de sensibilizar y comunicar a la sociedad momentos históricos, estéticos y culturales de nuestro contexto. Giovine Yáñez (2015) afirma que las artes comunican a través de las representaciones y, si la representaciones están vacías, no hay transmisión de sentido. Por ello es tan importante que durante la formación profesional artística no solo se vele por el desarrollo de una excelente técnica o competencias especializadas, sino también identificar el sentir de los futuros artistas. Pero ¿cómo aproximarse a las realidades que viven los estudiantes?

La investigación educativa es una de las herramientas que permite una aproximación con el estudiantado. No obstante, las investigaciones referentes al rendimiento, desempeño, procesos enseñanza aprendizaje, etc., llegan a ser menospreciadas o cuestionadas en el contexto universitario de las artes en México. Como lo menciona Tortajada (2008), el arte es un fenómeno misterioso que no puede ser analizado y, por tanto, "la investigación es un intruso incómodo en las escuelas especializadas de arte y en universidades donde se incluyen licenciaturas y posgrados, no se enfatiza la comprensión de la enseñanza ni las habilidades analíticas en la utilización del lenguaje y teoría" (p. 171).

En el mismo sentido, Torres (2016) plantea que, en los últimos años, por todos los continentes, educadores artísticos replantean los paradigmas de su campo teórico. Los investigadores están proponiendo debates sobre retos emergentes de la educación artística, como por ejemplo tecnologías electrónicas, maneras diferentes de comunicación en red, acercamientos, discursos alternativos sobre las artes y los públicos y nuevas concepciones. 
De igual forma, Lynton (2006) propone que en la danza es necesaria la investigación y la reflexión con la finalidad de divisar a las personas como una totalidad y no aislar lo cognitivo de lo afectivo y lo corporal o lo vivencial de lo conceptual. Menciona que las personas que se saben escuchadas y respetadas, escucharán y respetarán a los demás y que, al igual que con otros campos del saber y profesiones, la expresión artística forma parte de las experiencias que requieren de una preparación general integral que facilite en vez de perjudicar el interés y la motivación de los estudiantes para que puedan descubrir e intensificar sus estudios.

Retomando lo expuesto, Tortajada (2008) dice que la investigación artística puede ser, en lo general, aplicada o práctica, mientras su objetivo sea resolver problemas de la teoría, educación, creación o ejecución. Diversas investigaciones artísticas pueden inclinarse más por los procesos de creación y ejecución, pero también es necesario analizar factores del mismo proceso educativo y empezar a visualizar a las artes desde otras aristas.

Por tal motivo, este estudio busca un acercamiento con la comunidad estudiantil a través del sentido de pertenencia a fin de mejorar y reorientar procesos de enseñanza-aprendizaje que permitan, en un primer contacto, conocer su sentir respecto a su proceso formativo y, posteriormente, desarrollar experiencias más significativas y que abonen a una formación integral artística mucho más satisfactoria.

De acuerdo con Pino, Cavieres y Muñoz (2018), la permanencia exitosa de los estudiantes a lo largo de sus estudios universitarios ha adquirido gran importancia por las consecuencias positivas que trae para los propios estudiantes, sus familias y las instituciones de educación superior. Estos autores mencionan que el sentido de pertenencia emerge como un factor vinculado con aspectos personales e institucionales y, por tanto, permite una mirada integrada respecto a las variables que inciden en que los estudiantes desarrollen exitosamente sus estudios. Aunado a ello, el sentido de pertenencia puede potenciar los aprendizajes, mejorar el desempeño académico y las relaciones profesor-alumno-institución.

\section{Marco teórico: el sentido de pertenencia en el ámbito educativo}

La importancia del sentido de pertenencia en la vida del ser humano ha sido estudiada por diversos autores y abordada desde distintas perspectivas. Al revisar este concepto encontramos que, a lo largo de los años y de los contextos, el concepto ha mantenido características afines en sus acepciones. Dentro de las más repetidas se encuentra que el sentido de pertenencia es inherente 
al ser humano, como una necesidad. Los autores Baumeister y Leary (1995) determinaron que los seres humanos sienten una motivación intrínseca por pertenecer, afirman que las personas buscamos no solo tener relaciones interpersonales, sino también mantenerlas a largo plazo; explican que la motivación que tenemos por pertenecer comprende procesos cognitivos, patrones emocionales, comportamientos, salud y bienestar, lo que significa que el sentido de pertenencia es un proceso que permeará en todos los sentidos de nuestro desarrollo.

De manera coincidente, Morrison, Epstude y Roese (2012) indican que la necesidad de pertenecer es un núcleo central en el ser humano y que, cuando se satisface, se asocia potencialmente al bienestar y a la salud mental. En el mismo sentido, Mellor, Stokes, Firth, Hayashi y Cummins (2008) afirman que la necesidad de pertenecer cumple un rol fundamental en la vida de las personas al ser un precursor de las interacciones sociales.

En el caso de Hopenhayn y Sojo (2011), estos reconocen que el sentido de pertenencia es un factor que permite a las personas ejercer su vida dentro de una comunidad y que, al obtener un reconocimiento de los otros, los individuos logran definir sus proyectos de vida y llevarlos a cabo, lo que otorga un sentido de seguridad personal y fuerza colectiva.

Si sentirse parte de una comunidad contribuye con el desenvolvimiento personal, la escuela como comunidad juega un papel determinante en la vida de los estudiantes, no solo por el logro de metas personales, sino por el trabajo conjunto que se puede desarrollar si existe un sentimiento legítimo al grupo. No obstante, ¿las universidades o escuelas están preocupadas por mantener altos niveles de pertenencia? ¿O será que muchas veces se da por hecho que el sentido se desarrolla con el simple hecho de estudiar ahí?

Osterman (2000) expresa que si la escuela es capaz de entenderse como una comunidad, los miembros se sentirán queridos y apoyados, parte importante de la comunidad. En el caso de una escuela, se generaría un sentido de conexión compartido cargado de emotividad, en donde el aprendizaje se gestionaría desde la cooperación con los demás. Sin embargo, Osterman también menciona que es responsabilidad de la escuela y de los profesores fomentar el desarrollo del sentido de pertenencia, a través de actividades que involucren a toda la comunidad educativa.

En palabras de Guzmán y Saucedo (2007), al interior de las escuelas se dan relaciones explícitas e implícitas entre alumnos, y entre docentes y alumnos, que dan sentido al fenómeno educativo. Pero, en realidad, existen pocos acercamientos significativos entre los actores involucrados en las escuelas y esto tiene serias implicaciones que se ven reflejadas en algunas 
ocasiones en un profundo distanciamiento entre los intereses y necesidades reales de los estudiantes y las acciones realizadas por las autoridades educativas.

El sentido de pertenencia en el ámbito educativo puede ser un potenciador del quehacer artístico en las universidades, al empezar a atender muchos de los retos que presentan las artes, como lo señaló el Ministerio de Educación del Gobierno de España en su informe del 2010. Este informe plantea que la danza como oferta de la Educación Superior tiene desafíos a nivel internacional, por lo que es necesario demostrar que la danza es un instrumento para la acción e intervención socioeducativa, para mejorar la salud, elevar el espíritu y generar satisfacción y felicidad. El documento continúa diciendo que la danza debe abrirse nuevos caminos y que otro desafío es formar verdaderos artistas capaces de elaborar un discurso propio, con identidad creativa, con rigor y sensibilidad, que utilicen la técnica como un medio y no como un fin (Ministerio de Educación del Gobierno de España, 2010).

Si bien para lograr estos retos se requiere mucho más que un arraigado sentido de pertenencia, analizarlo permitiría detectar nichos de oportunidad para construir comunidades más comprometidas y motivadas a trabajar por el bien común. La comunidad en las artes juega un papel determinante para la consolidación de proyectos, puestas en escena y obras que dependen directamente de las relaciones interpersonales.

Al igual que Morrison, Epstude y Roese (2012) y Mellor et al. (2008), Brea (2014) coincide en que la necesidad de pertenecer a una comunidad o grupo está asociada con patrones emocionales de bienestar que conducen a emociones positivas como felicidad o alegría. Menciona también que, en terreno educativo, la experiencia del sentido de pertenencia se vincula con el desarrollo de procesos psicológicos claves para el éxito de los estudiantes, así como con actitudes positivas hacia el grupo social, hacia la academia y su entorno.

Huerta (2018) menciona que la identificación y satisfacción de las necesidades reales de cada individuo son directamente proporcionales a la percepción individual que este pueda tener o cómo lo pueda interpretar, pues si el individuo no siente o no percibe como atendidas estas necesidades reales, no podrá existir el vínculo que genere el compromiso intrínseco que caracteriza a la identidad con algo o con alguien; es decir, no se genera el sentido de pertenencia.

Brea (2014) junto con otros autores propone en lo general cuatro dimensiones que inciden o determinan el sentido de pertenencia con una institución educativa: la social-psicológica, la 
afectiva, la física y la académica. Esta investigación las retoma para analizar el sentido de pertenencia de los alumnos de la Licenciatura en Danza de la BUAP.

La dimensión social-psicológica, de acuerdo con Brea (2014), es la que significa en los alumnos sentirse seguros de sí mismos, valorados, necesitados y significativos dentro de un grupo o sistema. Asimismo, incluye los procesos de desarrollo de la competencia social que habilita a los seres humanos a interactuar significativamente con otros, a desarrollar lazos afectivos y construir relaciones perdurables que propiciaran integración grupal y cohesión social. En las artes esta dimensión es determinante en los procesos de creación e involucramiento de los montajes artísticos, ya que la funcionalidad de los grupos depende de la autopercepción positiva de cada integrante. Augé (1994) señala que la identidad individual se construye a través de relacionarse con los demás y compartir significados y experiencias entre ellos, de la misma manera que la identidad grupal se construye a partir de las interrelaciones grupales.

La dimensión afectiva se refiere a sentirse parte de un grupo o de una institución, y genera consigo alcances que permiten a los estudiantes entablar relaciones de fidelidad, confianza, sentimientos de identidad y de seguridad. Por tanto, fortalecer y garantizar el sentido de pertenencia es, en sí mismo, un fin. Para el caso específico de la danza, generar relaciones de confianza y afecto es vital para la vida profesional de los bailarines, debido a que las obras coreográficas requieren más allá de una vinculación física. Turner (1990) y Brea (2014) argumentan que el sentido de pertenencia se construye sobre la base de la identificación de la persona con el grupo, con el lugar y con la colectividad formal, cuyos valores y objetivos son conocidos y compartidos por el grupo.

La tercera dimensión es la física y, de acuerdo con Brea (2014), está constituida por la influencia del ambiente físico en su construcción. Esta dimensión la conforman los procesos de interacción conductual y simbólica que se verifican entre las personas y el ambiente físico. Se caracteriza por brindar ambientes agradables y estimulantes que ofrecen oportunidades para desarrollar habilidades y para construir experiencias memorables personales o colectivas. Muntañola (2004) explica que la identidad de lugar es una constante, porque define el encuentro entre el ambiente, el individuo y el grupo, por lo tanto, los lugares o espacios también son parte del sentido de pertenencia, así como las normas y valores acerca del espacio común y del espacio personal. 
Por último, Brea (2014) define la dimensión académica como la razón de ser de las universidades. Estas deben brindar a sus estudiantes una formación profesional bajo parámetros de calidad, principios éticos preestablecidos en su filosofía. Esta formación académica supone una estructura curricular y organizacional que coordine los contenidos y las estrategias didácticas dentro de un ambiente propicio al buen desenvolvimiento de los procesos de enseñanzaaprendizaje. Negrini (2010) sustenta que la conexión de los estudiantes con el centro educativo, sus profesores y sus pares, se asocia al logro académico, así como la desconexión se asocia al bajo rendimiento, problemas conductuales, apatía, ausentismo y deserción. Es decir que la conexión se convierte en una condicionante de la permanencia y la retención de los estudiantes y se relaciona con la vinculación, el apego y el compromiso de los estudiantes.

\section{Aspectos metodológicos}

El diseño del presente estudio es de tipo descriptivo no experimental, con enfoque cuantitativo, que busca describir y explicar los fenómenos a partir de información y datos cuantificables recabados en un tiempo determinado.

La población de la Licenciatura en Danza de la BUAP está conformada por 210 estudiantes, matrícula que se ha mantenido durante 2019-2020. La muestra que participó en este estudio fue de 108 alumnos de los diferentes semestres pares — segundo, cuarto, sexto, octavo y décimo-, semestres que responden al período escolar cuando se aplicó el instrumento (otoño 2019). La muestra estadística se extrae de una población con el criterio de que todos los miembros estén adecuadamente representados, resultando una muestra con un margen de error estadístico del $6.78 \%$ y un $95 \%$ de nivel de confianza.

\section{Instrumento utilizado}

El instrumento aplicado en este estudio es un cuestionario adaptado de Brea (2014). Su elección se debe a la propuesta que hace la autora referente a las cuatro dimensiones que determinan el sentido de pertenencia: la afectiva, social (psicológica), académica y física, que coinciden con los recursos y posturas de la universidad.

La adaptación del instrumento se realizó en primera instancia de manera contextual y por necesidades específicas de la licenciatura, por ejemplo, la importancia de la relación afectiva que se va generando con la universidad. La valoración de estas dimensiones se realizó a través de 
veinte indicadores (Figura 1) agrupados por bloques: ocho ítems de la dimensión afectiva, cuatro ítems de la dimensión social, cuatro de la académica y cuatro de la física. Los participantes debían responder a las afirmaciones de acuerdo con una escala tipo Likert con cuatro opciones como respuesta: nulo, a veces, casi siempre y siempre.

Cabe mencionar que además de estos 20 ítems el instrumento cuenta con un apartado de datos personales, académicos y sociodemográficos, tales como edad, sexo, semestre, promedio académico, así como si el alumno trabaja, pues se considera que estas variables permiten identificar el impacto del sentido de pertenencia.

\section{Validación}

Para la validación del instrumento se utilizaron diversos métodos. El primero de ellos fue presentar a un grupo de diez alumnos con alto promedio el instrumento acomodado en una sola hoja, para que no pareciera tedioso de contestar. Una vez contestado, se atendieron las dudas y se agregaron dos variables sociodemográficas que no se habían contemplado. Posteriormente, por medio del programa Statistical Package for the Social Sciences (SPSS) se realizó una prueba de consistencia interna, la cual sirvió para medir la fiabilidad de la escala dando como resultado un Alfa de Cronbach de .811 de los 20 ítems, lo que indica un alto grado de confiabilidad. 


\begin{tabular}{|c|c|c|c|c|c|c|}
\hline Dimensiones & & $\begin{array}{l}\text { Indicar con qué respuesta te identificas para las } \\
\text { siguientes afirmaciones: }\end{array}$ & 1. Nulo & $\begin{array}{l}\text { 2. Algunas } \\
\text { veces }\end{array}$ & $\begin{array}{l}\text { 3. Casi } \\
\text { siempre }\end{array}$ & 4. Siempre \\
\hline \multirow{8}{*}{ 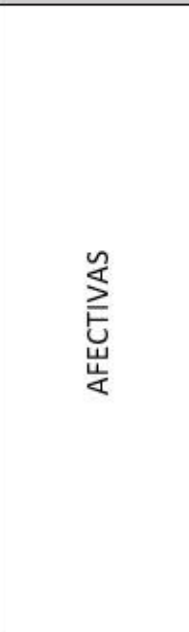 } & 1 & $\begin{array}{l}\text { Los profesores están disponibles fuera del horario de } \\
\text { clases para atender dudas y necesidades }\end{array}$ & & & & \\
\hline & 2 & $\begin{array}{l}\text { Los tutores brindan un seguimiento personalizado y } \\
\text { continuo a tu trayectoria }\end{array}$ & & & & \\
\hline & 3 & $\begin{array}{l}\text { La coordinación de la licenciatura atiende tus dudas y } \\
\text { necesidades }\end{array}$ & & & & \\
\hline & 4 & $\begin{array}{l}\text { Sientes afecto por el grupo de compañeros y docentes } \\
\text { con los que convives }\end{array}$ & & & & \\
\hline & 5 & Estás orgulloso de estudiar en la Licenciatura en Danza & & & & \\
\hline & 6 & Compartes la filosofía y los valores de la institución & & & & \\
\hline & 7 & Te sientes integrado en tu generación y sección & & & & \\
\hline & 8 & Estás orgulloso de pertenecer a la BUAP & & & & \\
\hline \multirow{4}{*}{ 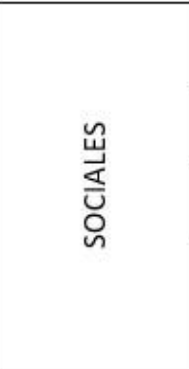 } & 9 & $\begin{array}{l}\text { Existe una motivación conjunta por parte de la } \\
\text { comunidad para alcanzar logros en común }\end{array}$ & & & & \\
\hline & 10 & Te sientes seguro de ti mismo en la Universidad & & & & \\
\hline & 11 & Se promueve el trabajo en equipo y el espíritu grupal & & & & \\
\hline & 12 & $\begin{array}{l}\text { Se promueve la interacción social con compañeros y } \\
\text { profesores dentro y fuera de la Universidad en } \\
\text { actividades extracurriculares o eventos artísticos }\end{array}$ & & & & \\
\hline \multirow{4}{*}{ 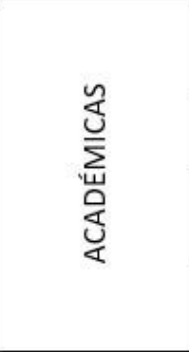 } & 13 & $\begin{array}{l}\text { Te sientes preparado y con los suficientes conocimientos } \\
\text { para poder desempeñarte en el ámbito laboral }\end{array}$ & & & & \\
\hline & 14 & $\begin{array}{l}\text { Los contenidos curriculares son de actualidad y tienen } \\
\text { aceptabilidad en el mundo real }\end{array}$ & & & & \\
\hline & 15 & $\begin{array}{l}\text { En el aula se respira un ambiente de disciplina y } \\
\text { cordialidad }\end{array}$ & & & & \\
\hline & 16 & $\begin{array}{l}\text { Existe compromiso académico por parte de los alumnos } \\
\text { y maestros para alcanzar las metas de cada asignatura }\end{array}$ & & & & \\
\hline \multirow{4}{*}{ 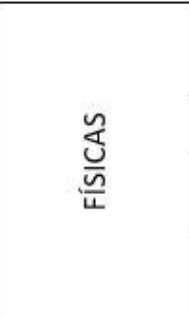 } & 17 & $\begin{array}{l}\text { Las clases prácticas y teóricas pueden realizarse } \\
\text { adecuadamente en los salones }\end{array}$ & & & & \\
\hline & 18 & Te sientes seguro dentro de la universidad & & & & \\
\hline & 19 & Las instalaciones se mantienen limpias & & & & \\
\hline & 20 & $\begin{array}{l}\text { Las instalaciones están en condiciones favorables para } \\
\text { desarrollar las actividades académicas }\end{array}$ & & & & \\
\hline
\end{tabular}

Figura 1. Instrumento dividido por dimensiones. Fuente: Elaboración propia (2020) 


\section{Composición de la muestra}

A continuación, se exponen los resultados más importantes de las de las variables académicas y sociodemográficas de los estudiantes encuestados. En la variable género, de los 108 alumnos, el $79 \%$ está representado por el género femenino y el $21 \%$ por el masculino. De manera regular en las diferentes generaciones de la licenciatura, la población femenina ha sido mayor que la masculina.

Las edades de los alumnos oscilan entre los 18 y 33 años, con una media de 21 años. Para un mejor manejo de la información obtenida, y debido a que $75 \%$ de la población está representado por edades de 18 a 22, se trabajó únicamente con dos grupos de edad: de 18 a 22 años y de 23 a 33 años. El primer grupo responde a estudiantes que mantienen una trayectoria académica continua y el segundo grupo una trayectoria discontinua.

En la distribución de alumnos por semestre se observa que el mayor porcentaje de participación del estudio se concentra en los primeros semestres - segundo semestre con 24.8\%, cuarto semestre con $30.5 \%$, sexto semestre con $14.3 \%$, octavo semestres con $17.1 \%$ y décimo semestre con $13.3 \%$ - lo cual permitirá seguir trabajando con estos mismos alumnos para estudios posteriores de seguimiento al sentido de pertenencia.

El rendimiento académico de los estudiantes indica que un $35 \%$ tienen un promedio mayor a 9, no obstante, se realizó un análisis de los estudiantes por el semestre que cursan en proporción a los rangos de promedio definidos para este estudio (Gráfico 2), en donde se puede observar que en los primeros semestres, segundo y cuarto, ninguno tiene un promedio menor a 7.6 y que en octavo y décimo semestre la mayoría obtiene un promedio arriba de ocho.

La última variable relacionada con el trabajo indica que 52\% de los alumnos participantes trabajan y el $48 \%$ no, lo cual representa que, al contar con más del 50\% de la población trabajado, esto podría representar una variable de mayor análisis para el estudio. 


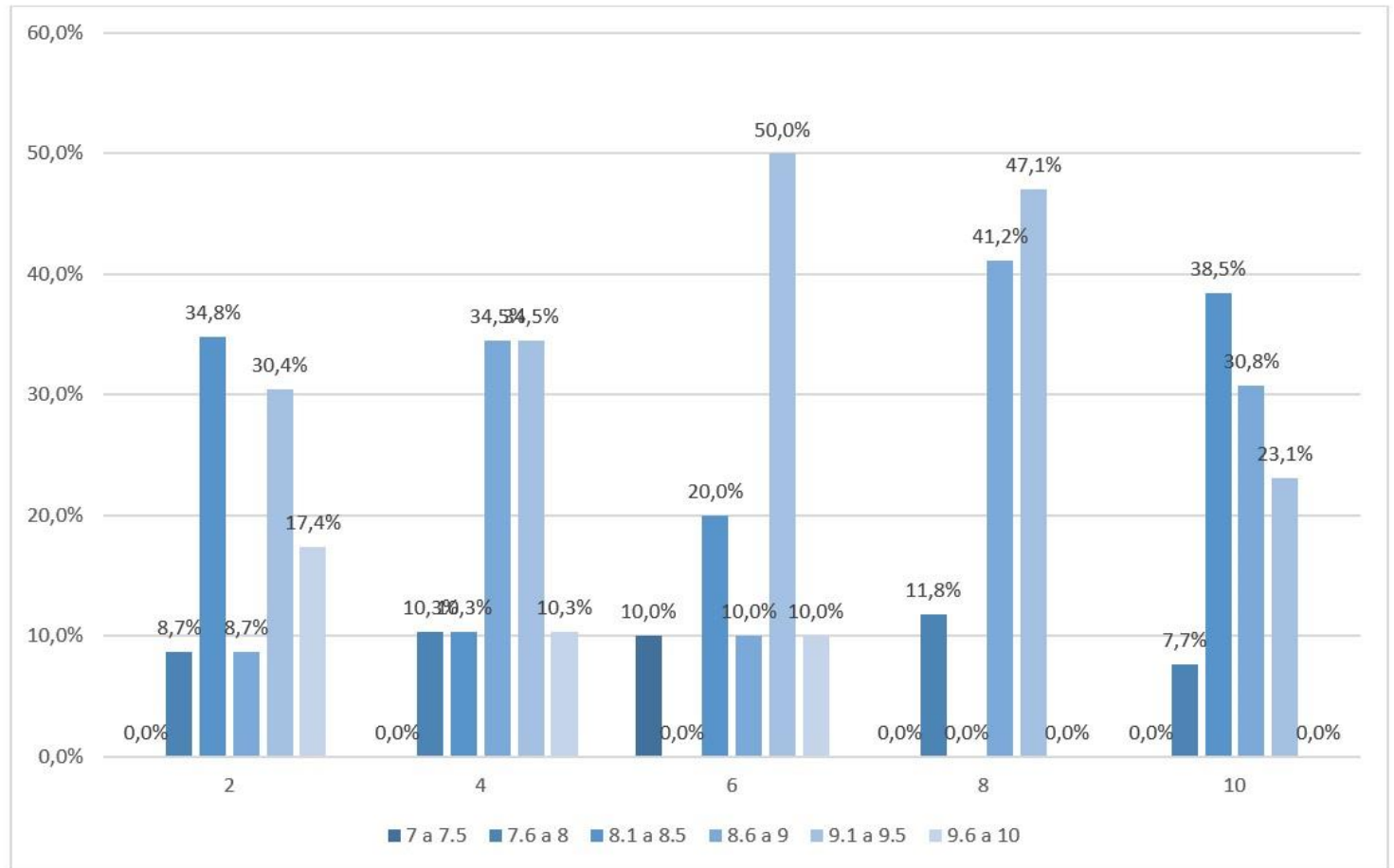

Gráfico 1. Rangos de promedio académico por semestre. Fuente: Elaboración propia (2020)

\section{Resultados y discusión}

Para analizar cada una de las dimensiones de sentido de pertenencia se realizó una estandarización de dichas variables, con la finalidad de poder hacer comparaciones y conocer su comportamiento. Al analizar las medias y desviación estándar de cada dimensión mediante el programa SPSS se puede observar en la Tabla 1 que la dimensión social y académica son las que cuentan con una valoración inferior, con 52.39 y 53.24 respectivamente.

Uno de los factores determinantes para analizar la percepción de los estudiantes es la desviación estándar y, como se puede apreciar, en la dimensión social es mayor (34.60), lo que significa que la opinión de los estudiantes es más dispersa respecto a la dimensión académica (19.50). Es decir que la percepción de esta última mantiene mayor cercanía de opinión, por lo que la dimensión académica sería la que cuenta con una menor valoración por parte de los alumnos de las cuatro dimensiones. 
Tabla 1.

Medias por dimensión estandarizada

\begin{tabular}{lcc}
\hline & Media & $\begin{array}{c}\text { Desviación } \\
\text { estándar }\end{array}$ \\
\hline $\begin{array}{l}\text { Dimensión afectiva } \\
\text { estandarizada }\end{array}$ & 63,70 & 17,50 \\
\hline $\begin{array}{l}\text { Dimensión social } \\
\text { estandarizada }\end{array}$ & 50,08 & 23,25 \\
\hline $\begin{array}{l}\text { Dimensión académica } \\
\text { estandarizada }\end{array}$ & 53,24 & 19,50 \\
\hline $\begin{array}{l}\text { Dimensión física } \\
\text { estandarizada }\end{array}$ & 70,99 & 18,88 \\
\hline
\end{tabular}

Fuente: Elaboración propia (2020)

Cabe recordar que la dimensión académica se asocia con los resultados productivos de los estudiantes, en rendimiento, persistencia y retención. La ausencia de esta dimensión, siguiendo a Brea (2014) y Negrini (2010), suele conducir a una disminución del interés y del compromiso con las actividades ordinarias de la vida académica. Estos autores afirman que cuando esta necesidad no está satisfecha, la motivación de los estudiantes disminuye y la dificultad para comprometerse incrementa.

La dimensión académica también engloba la seguridad y confianza que siente el alumno respecto a los conocimientos prácticos y teóricos que le brinda la universidad a través del plan de estudios, su modelo académico y el proceso de enseñanza-aprendizaje que desarrolla con sus profesores. En este sentido es importante señalar que esta dimensión representa un área de oportunidad para la Licenciatura en Danza dado que, a través de los resultados obtenidos, se observa que los alumnos están medianamente satisfechos con los conocimientos adquiridos para desempeñarse en el ámbito laboral y, a su vez, cuestionan la pertinencia de los contenidos curriculares del plan de estudios. Es oportuno mencionar que la última actualización del plan de estudios se realizó en 2016. Los alumnos que están cursando este plan de estudios son alumnos de segundo, cuarto y sexto semestre, mientras que los de octavo y décimo semestre cursan un plan de 
estudios elaborado en el 2009 es decir que, a pesar de la actualización curricular, los alumnos perciben un bajo nivel de pertinencia. Esta dimensión permite identificar la necesidad de establecer un mecanismo de comunicación que difunda con profesores y alumnos las actualizaciones curriculares de los últimos años con la finalidad reforzar el logro de objetivos de cada asignatura.

La dimensión social-psicológica, con una media de 52.39 y una desviación estándar de 34.60, indica que es la dimensión con mayor dispersión en los datos, lo que significa que la variabilidad de opiniones de los estudiantes está repercutiendo negativamente en la seguridad que tienen en sí mismos y para desenvolverse con los demás.

La satisfacción de la dimensión social-psicológica de los estudiantes, de acuerdo con Osterman (2000), influye en el desarrollo de importantes procesos psicológicos como lo son la motivación intrínseca, internalización, el bienestar y la salud. Una persona automotivada buscará retos y oportunidades de expandir sus conocimientos y sus experiencias, mientras que los procesos de aceptación de normas, valores y regulaciones apoyan el desarrollo de la autonomía y la resiliencia. Esto quiere decir que el trabajo interpersonal que se hace con los estudiantes desde la licenciatura aún no es suficiente para consolidar su autonomía y motivación personal.

La dimensión afectiva, que obtuvo una media del 63.70 y una desviación estándar de 17.50, representa una menor desviación estándar que el resto, lo que supone una mayor homogeneidad en la opinión de los estudiantes. Las dimensiones social-psicológica y afectiva están íntimamente relacionadas y pueden llegar a confundirse, por la relación que guardan los individuos con el grupo. La diferencia radica en que la primera habla de la seguridad del individuo ante el grupo al que pertenece, haciendo que la iniciativa y la seguridad personal se fortalezca en el trabajo en conjunto, y en el caso de la dimensión afectiva se refiere a los lazos de confianza que generan entre la universidad, la facultad y la estructura organizacional que acoge a los alumnos, por lo que se puede sentar que las relaciones afectivas tienen un mayor impacto en la memoria colectiva institucional, al sentirse los estudiantes orgullosos por ser parte de una universidad reconocida a nivel nacional e internacional.

Brea (2014) afirma que esta memoria colectiva constituye un elemento de cohesión y diferenciación tan fuerte y poderoso que se superpone a situaciones de amenaza de extinción o vulnerabilidad grupal o institucional. Se convierte así en el principal aliado de permanencia y continuidad de los estudiantes. En este sentido, los estudiantes de la licenciatura en danza reflejan un estatus que permite el desarrollo de esta comunidad. 
Por último, con una media de 70.99 y una desviación estándar de 18.88, se visualiza que la dimensión física es la mejor valorada por los estudiantes. Los espacios universitarios y los propios de la Facultad de Artes son percibidos como esferas donde se sienten seguros, pueden experimentar y compartir durante el proceso de enseñanza-aprendizaje.

Una de las particularidades de las licenciaturas en danza es que los alumnos permanecen mucho más tiempo en las aulas que un alumno de otra área. Esto se debe a la exigencia que demanda la disciplina, como el fortalecimiento corporal que requiere mantener, ensayos de puestas en escena, montajes extras a las clases, desarrollo de propuestas coreográficas, entre otras, por lo que los estudiantes pasan más de ocho horas entre clases y actividades extras.

Es evidente que la dimensión física se vuelve indispensable para la vida académica; lo sustancial es que a partir de esta se pueden diseñar estrategias que no solo hagan sentirse cómodos y seguros a los alumnos dentro de la Facultad de Artes, sino que los espacios sean usados como generadores de experiencias estéticas y de aprendizaje que permitan fortalecer las demás dimensiones. Tal como lo menciona Turner (1990), la pertenencia se construye sobre la base de la identificación de la persona con el grupo, con el lugar y con la colectividad.

Si bien este análisis reveló cómo se percibe cada una de las dimensiones que determinan el sentido de pertenencia, en el análisis descriptivo se observa que algunas de las variables independientes podrían presentar diferencias que valiera la pena analizar. Una es el género, debido a que la población femenina supera por mucho a la población masculina. De manera histórica en el programa educativo el género femenino ha prevalecido en matrícula. Por tal motivo se procedió a analizar a estos dos grupos a través de la prueba no paramétrica de Kolmogórov-Smirnov en SPSS, determinando que los datos tienen un comportamiento no normal.

Para observar si existían diferencias significativas entre estos dos grupos se realizó la prueba de U de Mann-Whitney y se obtuvo como resultado que no existen diferencias significativas entre género para ninguna de las dimensiones. Para la dimensión afectiva se obtuvo un .527 sig., para la dimensión social un .515 sig., dimensión académica un .561 sig., y para la dimensión física un .050 sig. Esto significa que las cuatro dimensiones no se verán afectadas por el género, no importa si son hombres o mujeres no representan grupos que tengan que ser trabajados de manera particular para lograr un mayor de sentido de pertenencia, es decir que en las acciones que se implementen no tendrán que ser diferenciadas por el género. 
La variable de trabajo supone diferencias de percepción entre los alumnos que trabajan y los que no; por tal motivo se efectuó la misma prueba y se encontró que únicamente existe diferencia significativa en la dimensión social (Figura 2). Esta dimensión simboliza la autonomía, el autoconcepto y la seguridad en sí mismos respecto a su interacción social. Las diferencias entre el grupo de alumnos que trabaja y el que no significan que los alumnos que trabajan, al entablar otro tipo de relaciones como las laborales, ven su autopercepción y autoconfianza más afianzada.

En cuanto a las dimensiones afectiva, académica y física, el que los alumnos trabajen o no lo hagan no afecta la percepción de estas variables. Sin embargo, las estrategias de autoconfianza deben estar mayormente dirigidas a con los alumnos que no trabajan para incrementar el sentido de pertenencia.

\section{Resumen de contrastes de hipótesis}

\begin{tabular}{|c|c|c|c|c|}
\hline & Hipótesis nula & Prueba & Sig. & Decisión \\
\hline 1 & $\begin{array}{l}\text { La distribución de Dimensión } \\
\text { Afectiva Estandarizada es la } \\
\text { misma entre las categorías de } \\
\text { Trabajas (SI-NO). }\end{array}$ & $\begin{array}{l}\text { Prueba U de } \\
\text { Mann-Whitney } \\
\text { para muestras } \\
\text { independiente } \\
\text { s }\end{array}$ & .070 & $\begin{array}{l}\text { Conserve la } \\
\text { hipótesis } \\
\text { nula. }\end{array}$ \\
\hline 2 & $\begin{array}{l}\text { La distribución de Dimensión } \\
\text { Social Estandarizada es la misma } \\
\text { entre las categorías de Trabajas } \\
\text { (SI-NO). }\end{array}$ & $\begin{array}{l}\text { Prueba U de } \\
\text { Mann-Whitney } \\
\text { para muestras } \\
\text { independiente } \\
\text { s }\end{array}$ & .018 & $\begin{array}{l}\text { Rechace la } \\
\text { hipótesis } \\
\text { nula. }\end{array}$ \\
\hline 3 & $\begin{array}{l}\text { La distribución de Dimensión } \\
\text { Académica Estandarizada es la } \\
\text { misma entre las categorías de } \\
\text { Trabajas (SI-NO). }\end{array}$ & $\begin{array}{l}\text { Prueba U de } \\
\text { Mann-Whitney } \\
\text { para muestras } \\
\text { independiente } \\
\mathrm{s}\end{array}$ & .111 & $\begin{array}{l}\text { Conserve la } \\
\text { hipótesis } \\
\text { nula. }\end{array}$ \\
\hline 4 & $\begin{array}{l}\text { La distribución de Dimensión } \\
\text { Física Estandarizada es la misma } \\
\text { entre las categorías de Trabajas } \\
\text { (SI-NO). }\end{array}$ & $\begin{array}{l}\text { Prueba U de } \\
\text { Mann-Whitney } \\
\text { para muestras } \\
\text { independiente } \\
\text { s }\end{array}$ & .249 & $\begin{array}{l}\text { Conserve la } \\
\text { hipótesis } \\
\text { nula. }\end{array}$ \\
\hline
\end{tabular}

Se muestran significaciones asintóticas. El nivel de significancia es .05 .

Figura 2. Prueba de U de Mann-Whitney SPSS-Variable Trabajo.

Fuente: Elaboración propia (2020)

El semestre que cursan los estudiantes también se sometió a una prueba no paramétrica a través de la prueba Kruskal-Wallis que sirve para comparar a más de dos grupos (Figura 3). Los resultados obtenidos confirman que existen diferencias significativas entre los semestres respecto a las cuatro dimensiones de sentido de pertenencia. 
A partir de un análisis de medianas se pudo distinguir que los primeros semestres tienen una mejor percepción de las cuatro dimensiones y que, conforme el semestre va incrementando, este sentido de pertenencia va disminuyendo. Esto resultó un hallazgo que requiere de atención a corto plazo. Los alumnos de semestres más adelantados representan una guía y ejemplo para los alumnos de los primeros semestres en diversos sentidos: los líderes de grupos artísticos suelen ser de los últimos semestres, los mentores académicos se seleccionan a partir del sexto semestre y estos guían en procesos administrativos, académicos y de servicios de apoyo al estudiante. Esto representa para la licenciatura un riesgo de identidad y unidad.

Los resultados arrojaron que el semestre es una variable determinante para valorar el sentido de pertenencia, encontrando que los alumnos de los últimos semestres no se sienten con los conocimientos suficientes para desenvolverse fuera de la universidad. Si bien este puede ser un sentimiento por el que atraviesen muchos egresados, es importante señalar que debido a que la satisfacción va disminuyendo semestre a semestre, es necesario establecer estrategias que robustezcan el área curricular.

Estas acciones pueden encaminarse a fortalecer principalmente la dimensión académica con los estudiantes de los últimos semestres, puesto que esta dimensión aborda la congruencia del plan de estudios, el acercamiento con el medio laboral, además de la seguridad que tienen los alumnos acerca de sus propios conocimientos en danza, mientras que, con los primeros semestres, el trabajo se centraría en la dimensión social y posteriormente en la académica.

Con base en estos resultados, se pudo observar que la motivación es uno de los elementos que debe trabajarse transversalmente en las cuatro dimensiones. Freeman, Anderman y Jensen (2007) explican que el sentido de pertenencia se asocia significativamente a tres factores motivacionales: la valoración por las tareas académicas — que los alumnos perciban que las tareas o trabajos sean útiles para sus formación profesional-, la autoeficacia académica — que los alumnos en un ideal deberían tener confianza en las propias habilidades para completar las tareas asignadas - y la motivación intrínseca — que los alumnos disfruten realizar los trabajos y actividades.

Especialmente en la danza, la carga física y emocional es elevada debido a las demandas técnicas y creativas que se requieren. En muchos de los casos estas actividades, que podrían ser considerarse como motivadoras, se han llegado a percibir como actividades de estrés y molestia en los estudiantes, por lo que sería importante analizar y mejorar la motivación en ellos. 
Para desarrollar estos factores motivacionales es necesario iniciar también un análisis del sentido de pertenencia con la planta docente. Pino, Cavieres y Muñoz (2018) mencionan que la relación con los profesores y el sentido de pertenencia ya está definida, será necesario verificar la relación entre las características de la enseñanza, motivación de los estudiantes y su sentido de pertenencia. Estos análisis son especialmente relevantes en países en los que este tipo de estudios son escasos en las artes.

\begin{tabular}{|c|c|c|c|c|}
\hline & Resumen de con & trastes de hip & tesis & \\
\hline & Hipótes is nula & Prueba & Sig. & Decisión \\
\hline 1 & $\begin{array}{l}\text { La distribución de Dimensión } \\
\text { Afectiva Estandarizada es la } \\
\text { misma entre las categorias de } \\
\text { Trabajas (SI-NO). }\end{array}$ & $\begin{array}{l}\text { Prueba U de } \\
\text { Mann-Whitney } \\
\text { para muestras } \\
\text { independiente } \\
\mathrm{s}\end{array}$ & .070 & $\begin{array}{l}\text { Conserve la } \\
\text { hipótesis } \\
\text { nula. }\end{array}$ \\
\hline 2 & $\begin{array}{l}\text { La distribución de Dimensión } \\
\text { Social Estandarizada es la misma } \\
\text { entre las categorías de Trabajas } \\
\text { (SI-NO). }\end{array}$ & $\begin{array}{l}\text { Prueba U de } \\
\text { Mann-Whitney } \\
\text { para muestras } \\
\text { independiente } \\
\mathrm{s}\end{array}$ & .018 & $\begin{array}{l}\text { Rechace la } \\
\text { hipótesis } \\
\text { nula. }\end{array}$ \\
\hline 3 & $\begin{array}{l}\text { La distribución de Dimensión } \\
\text { Académica Estandarizada es la } \\
\text { misma entre las categorías de } \\
\text { Trabajas (SI-NO). }\end{array}$ & $\begin{array}{l}\text { Prueba U de } \\
\text { Mann-Whitney } \\
\text { para muestras } \\
\text { independiente } \\
\mathrm{s}\end{array}$ & .111 & $\begin{array}{l}\text { Conserve la } \\
\text { hipótesis } \\
\text { nula. }\end{array}$ \\
\hline 4 & $\begin{array}{l}\text { La distribución de Dimensión } \\
\text { Física Estandarizada es la misma } \\
\text { entre las categorías de Trabajas } \\
\text { (SI-NO). }\end{array}$ & $\begin{array}{l}\text { Prueba U de } \\
\text { Mann-Whitney } \\
\text { para muestras } \\
\text { independiente } \\
\mathrm{s}\end{array}$ & .249 & $\begin{array}{l}\text { Conserve la } \\
\text { hipótesis } \\
\text { nula. }\end{array}$ \\
\hline & Se muestran significaciones asintótic & cas. El nivel de si & nificanci & ia es .05 . \\
\hline
\end{tabular}

\section{Conclusiones}

Conocer cómo se perciben las dimensiones que integran el sentido de pertenencia resulta enriquecedor para identificar áreas de oportunidad y fortalezas en las que se puede intervenir a corto, mediano y largo plazo en la Licenciatura en Danza de la BUAP. Integrar este tipo de estudios se vuelve fundamental para las escuelas de artes en una época donde los planteamientos teóricos y tecnológicos evolucionan rápidamente, obligando a los estudiantes a sentir más, conocer más y hacer más desde la danza y las artes. Si bien esto responde a demandas económicas, sociales y políticas, los formadores deben considerar la integración y diversificación de herramientas que 
puedan aportar a la trayectoria académica y la inserción laboral. El desconocimiento del sentir estudiantil durante su formación profesional limitará sin duda los resultados académicos y sociales que se esperan de estos.

El sentido de pertenencia señala indicadores puntuales que pueden motivar o desmotivar el transcurso de los estudiantes durante su formación. Manejar el mismo sistema de creencias entre la comunidad universitaria ayudará a consolidar los objetivos institucionales y curriculares; en palabras de Pino, Cavieres y Muñoz (2018), un buen sentido de pertenencia podría ayudar a los alumnos a completar sus estudios, pero la falta de este también podría limitar las interacciones que se establecen a lo largo de sus años de estudio, restringiendo igualmente su comprensión respecto a qué significaría formarse exitosamente para su futura vida profesional.

En este estudio se pudo observar que el sentido de pertenencia de los estudiantes con la licenciatura aún tiene tareas por atender. En relación con la dimensión académica se puede concluir que existe una brecha por cerrar conforme a la opinión estudiantil, y es que la formación que están recibiendo aún no alcanzan el nivel que ellos esperan para poder desarrollarse con confianza en el mundo laboral. Esto responde principalmente a dos factores: la falta de compromiso de los profesores por cumplir con los contenidos temáticos y la pertinencia de estos contenidos en correspondencia con las tendencias de la danza a nivel internacional.

Esto representa un pendiente que debe priorizarse mediante las siguientes propuestas: a corto plazo, la sensibilización con los profesores y la realización de un estudio de pertenencia que también los caracterice, a fin de comparar posturas y buscar soluciones más enfocadas en los procesos enseñanza-aprendizaje; a mediano plazo, buscar la actualización disciplinar y pedagógica de los profesores para mejorar las clases y asegurar que los contenidos respondan al contexto que guía a la danza en un mundo que evoluciona cada vez más rápido.

En torno a la dimensión social-psicológica, se refiere la necesidad de promover con los estudiantes mayor sentido de autoconfianza y del vínculo que pueden generar con la comunidad de la licenciatura. Si bien la dimensión social y afectiva son distintas, a partir de este estudio se pudo constatar que para este programa educativo deben trabajarse de manera paralela para poder crear verdaderos vínculos afectivos entre la comunidad. El sentimiento de percibirse parte de un grupo y la autoconfianza van acompañados de la valoración de los otros. En la danza este reconocimiento se puede trabajar a partir de la creación de vínculos más cercanos incorporado propuestas y procesos creativos dirigidos y creados por los mismos estudiantes. 
Se propone a corto y mediano plazo la organización de actividades extracurriculares orientadas al trabajo en equipo, conformación de grupos estudiantiles con responsabilidad social y ambiental, generación de propuestas artísticas en donde participen alumnos de los distintos semestres y profesores, hacer mayor difusión de las propuestas y proyectos que generen los estudiantes e incrementar los foros que permitan el diálogo en cuanto temas dancísticos y de autoconfianza.

Respecto a la dimensión física, se puede concluir que es la mejor valorado por los estudiantes. La apropiación positiva de los espacios facilitará el desarrollo de los vínculos afectivos, la comodidad estructural para trabajar afianza un trabajo conjunto. Será importante seguir fortaleciendo con profesores y alumnos que las aulas y los espacios comunes sean lugares donde se reaniman las relaciones interpersonales, donde se generan ambientes de aprendizaje y se fomente el respeto y compañerismo, haciéndolos participes del cuidado y mantenimiento de las instalaciones.

A modo de recomendación, realizar estudios de sentido de pertenencia en los centros de artes permitirá distinguir aciertos y áreas de oportunidad con la finalidad de consolidar una mejor formación integral. Darle voz al estudiantado abre una ventana de posibilidades a optimizar la calidad de las relaciones intra e interpersonales. Como lo mencionan Haussmann, Schofield y Woods (2007), las percepciones de los mismos alumnos vinculan sus experiencias con factores personales referidos a su contexto afectivo y social, así como factores institucionales que dan cuenta de su interacción con actores relevantes del contexto académico en el cual se desarrollan.

El constructo teórico resultante posibilita no solo una mejor comprensión de por qué los estudiantes deciden permanecer en sus programas de estudios al sentirse parte de las instituciones que los imparten, sino que también sugiere la importancia de los contextos en los cuales los estudiantes desarrollan su vida académica y el cómo contribuyen a que completen con éxito sus estudios (Haussmann, Schofield y Woods, 2007, p.839)

Cuando los alumnos posean sentido de pertenencia con su universidad y programa educativo se producirá un mejor rendimiento y entendimiento académico, se generarán sentimientos de autoconfianza y logro de objetivos conjuntos, teniendo como principal resultado la fácil integración de los estudiantes con la sociedad, promoviendo de este modo que la sociedad también tenga un mayor sentido con las artes. 


\section{Referencias}

Baumeister, R., y Leary, M. (1995). The need to belong: Desire for interpersonal Attachments as a Fundamental Human Motivation. Psychological Bulletin, 117(3). Recuperado de https://www.researchgate.net/publication/15420847_The_Need_to_Belong_Desire_for_Interpersonal_Atta chments_as_a_Fundamental_Human_Motivation

Brea, L. (2014). Factores determinantes del sentido de pertenencia de los estudiantes de Arquitectura de la Pontificia Universidad Católica Madre y Maestra, Campus Santo Tomás de Aquino (Tesis de doctorado). Recuperado de https://www.tdx.cat/bitstream/handle/10803/284952/TLMBA.pdf?sequence=1

Freeman, T., Anderman L., y Jensen J. (2007). Sense of belonging in college freshmen at the classroom and campus levels. The Journal of Experimental Education, 75(3), 203-220.

Giovine Yáñez, M. A. (2015). Paradojas del arte contemporáneo. Tópicos del Seminario, (34),155-171. Recuperado de https://www.redalyc.org/articulo.oa?id=594/59443297008

Guzmán, C., y Saucedo, C. (2007). La voz de los estudiantes: experiencias en torno a la escuela. Barcelona, España: Ediciones Pomares.

Haussmann, L., Schofield, J., y Woods, R. (2007). Sense of belonging as a predictor of intentions to persist among African American and white first-year college students. Research in Higher Education, 48(7), 803-839.

Hopenhayn, M., y Sojo, A. (Eds.). (2011). Sentido de Pertenencia en sociedades fragmentadas: America Latina en una perspectiva global. Buenos Aires, Argentina: Siglo XXI.

Huerta, A. (2018). El sentido de pertenencia y la identidad como determinante de la conducta, una perspectiva desde el pensamiento complejo. IE Revista de investigación educativa de la REDIECH, 9(16), 83-97.

Lynton, A. (2006). Crear con el movimiento: la danza como proceso de investigación. REencuentro. Análisis de Problemas Universitarios, (46), 51-57.

Mellor, D., Stokes, M., Firth, L., Hayashi, Y., y Cummins, R. (2008). Need for belonging, relationship satisfaction, loneliness, and life satisfaction. Personality and Individual Differences, 45(3), 213-218. https://doi.org/10.1016/j.paid.2008.03.020

Ministerio de Educación del Gobierno de España. (2010). Informe anual sobre el estado y situación de las Enseñanzas Artísticas. Recuperado

de https://sede.educacion.gob.es/publiventa/descarga.action?f_codigo_agc=14678_19

Muntañola, J. (2004). Arquitectura, educación y dialogía social. Revista Española de Pedagogía, 62(228), $221-228$. Recuperado de https://revistadepedagogia.org/lxii/no-228/arquitectura-educacion-y-dialogiasocial/101400010502/

Morrison, M., Epstude, K., y Roese, N. (2012). Life regrets and the need to belong. Social Psychological and Personality Science, 3(6), 1-7. https://doi.org/10.1177/1948550611435137

Negrini, I. (2010). Involucramiento de las estudiantes y los estudiantes en el proceso educativo. Revista Iberoamericana sobre Calidad, Eficacia y Cambio en Educación, 8(1), 63-78.

Osterman, K. (2000). Students' Need for Belonging in the School Community. Review of Educational Research, 70(3), 323-367. https://doi.org/10.3102/00346543070003323 
Pino, T., Cavieres, E., y Muñoz, J. (2018). Los factores personales e institucionales en el sentido de pertenencia de estudiantes chilenos a lo largo de sus estudios superiores. Revista Iberoamericana de Educación Superior, 9(25), 24-41. Recuperado de https://www.redalyc.org/articulo.oa?id=2991/299158443002

Tortajada, M. (2008). La investigación artística mexicana en el siglo XX. Cultura y Representaciones Sociales, 2(4), 169-196.

Torres, T. (2106). Del arte por el arte a las artes comprometidas con las comunidades: paradigmas actuales entre educación y artes. Pensamiento, Palabra y Obra, 16, 14-23. Recuperado de http://www.scielo.org.co/pdf/ppo/n16/n16a03.pdf

Turner, J. (1990). Redescubrir el grupo social. Una teoría de la categorización del Yo. Madrid, España: Ediciones Montara. Recuperado de https://sociopsicologia.files.wordpress.com/2010/10/una-teoria-de-lacategorizacion-del-yo-turner.pdf

\section{Contribución autoral}

a) Concepción y diseño del trabajo; b) Adquisición de datos; c) Análisis e interpretación de datos; d) Redacción del manuscrito; e) revisión crítica del manuscrito.

A. C. ha contribuido en a, b, c, d, e.

Editora científica responsable: Mag. Florencia de León 